\title{
PLASMA SHEATH STRUCTURE FOR DIFFERENT TEMPERATURE OF IN-STREAMING PARTICLES
}

\author{
S. K. Bhattarai*, R. Joshi* and R. Khanal* \\ *Central Department of Physics, Tribhuvan University, Kirtipur, Kathmandu, Nepal.
}

\begin{abstract}
Abstra ct: The structure of plasma sheath formed in front of an absorbing material wall for different temperatures of the in-streaming particles has been studied. For given electron and ion distributions at the sheath entrance we use the Kinetic Trajectory Simulation model to obtain the solution to a non-neutral, collisionless plasma sheath. Starting from an initial guess, the potential profile is iterated and our numerical method provides the final time-independent, selfconsistent state. It is observed that the sheath structure is highly influenced by varying the temperature.
\end{abstract}

Key words: Plasma; Quasineutrality; Debye shielding; Sheath; Presheath; Bohm criterion.

\section{INTRODUCTION}

In all practical plasma devices plasma interacts with the vessel (material walls). The interaction of plasma with the wall has been a matter of interest in all applications of plasma including the schemes to harness the fusion energy.

Plasma contains ions as well as electrons but the mobility of the electrons is much greater and hence can reach the wall earlier than the ions. This makes the wall negatively charged. Due to this, further electrons going towards the wall are repelled whereas the ions are accelerated towards the wall. Thus near the wall, the electron density decreases much faster than that of ions and a positive space charge region is formed shielding the potential at the wall. This shielding is of the order of some electron Debye length. This space charge region of positive ions which is very short, compared to the characteristics plasma length, is called the "Sheath". The sheath structure formed adjacent to a wall facing plasma contributes to the stability of the core plasma. Because of the sheath formed the negative potential at the wall can not penetrate deep into the core plasma and the core is virtually undisturbed.

For a sheath to exist, the in-streaming ions at the sheath entrance have to satisfy a condition called the "Bohm criterion" [1] [2] [3]. In it's kinetic form, this criterion reads,

$$
\left\langle\frac{1}{v^{2}}\right\rangle \leq \frac{m^{i}}{k\left(\gamma^{i} T_{p s}^{i}+\gamma^{e} T_{p s}^{e}\right)}
$$

where $k$ is the Boltzmann constant, $\gamma^{i}$ and $y^{e}$ are the ion and electron polytropic constants, respectively, and $T_{p s}^{i}$ and $T_{p s}^{e}$ are the ion and electron temperatures at the presheath side of sheath edge, respectively. The condition (1) ensures the potential profile to be non-oscillatory at the sheath edge. In realistic cases, it is usually satisfied marginally, i.e., with the equality sign.

The Bohm criterion implies that we need an electric field in the region preceding the sheath in order to accelerate the ions. The region in the plasma where the acceleration of the ion begins is called the presheath. Thus the plasma confined in a vessel can generally be classified into three regions: the sheath, the presheath and the bulk plasma.

It is important to note that the sheath structure is also responsible for determining the energy and particle flow towards the wall and overall bulk plasma behavior. In the sheath region the plasma is significantly non-neutral although it is practically quasineutral at the sheath entrance and in the presheath region as well. As the wall is negatively charged even the Debye shielding cannot counteract the potential due to the wall and a residue potential of the order $k T_{e} / m$ can always leak out from the sheath which is the primary cause of ion acceleration towards the sheath from presheath region. Hence, a very small potential difference occurs in the presheath region, which is very large in terms of length compared to the sheath. This helps in sheath formation provided the ion velocity satisfies the Bohm criterion.

In the present work, we have studied a space charge sheath formation, for different values of the presheath ion temperature, adjacent to an absorbing wall with presheath plasma on the other side, which we assume to be described by a two-fluid solution. We use the Kinetic Trajectory Simulation (KTS) model to obtain the solution to a nonneutral, time-independent, collisionless plasma sheath. It has been observed that the sheath structure is highly influenced by varying the temperature.

Awthor for Correspondeno: Raju Khanal, Central Department of Physics, Tribhuvan University, Kirtipur, Kathmandı, Nepal. Email: plasmanepal@hotmail.com. 


\section{BASIC PRINCIPLE OF KTS}

The characteristic feature of the KTS method is that the distribution functions of the particle species involved are calculated directly by solving the related kinetic equations along the respective collisionless particle trajectory [4] [5]. In order to obtain the distribution function at any point $[x, v]$ of the phase-space we trace the related trajectories to some phase-space where the distribution is given. We assume the electron and ion velocity distribution functions at the sheath edge to be cut-off Maxwellian in such a way that the most important requirements of the presheath-sheath transition are satisfied, i.e. quasi-neutrality, the sheath-edge singularity condition, continuity of the first three moments of each species, and the kinetic Bohm criterion.

In the general case of time-dependent, collisional kinetic theory, the species-s velocity distribution function $f^{s}(\vec{x}, \vec{v}, t)$ satisfies the kinetic equation

$\frac{d_{s} f^{s}}{d t}=\left(\frac{\partial}{\partial t}+\vec{v} \cdot \frac{\partial}{\partial \vec{x}}+\vec{a}^{s} \cdot \frac{\partial}{\partial \vec{v}}\right) f^{s}=c^{s}$

with

$$
\vec{a}^{s}(\vec{x}, \vec{v}, t)=\frac{q^{s}}{m^{s}}[\vec{E}(\vec{x}, t)+\vec{v} \times \vec{B}(\vec{x}, t)]
$$

Here $\vec{E}(\vec{x}, t)$ and $\vec{B}(\vec{x}, t)$ are the macroscopic (i.e. locally averaged) electromagnetic fields, $\vec{a}^{s}$ is the macroscopic acceleration of a species-s particles. (i.e. it's acceleration in these fields), and $c$ is the species-s collision term.

For collisionless cases the kinetic equation (2) takes the well known form of Vlasov equation

$$
\left(\frac{\partial}{\partial x}+\vec{v} \cdot \frac{\partial}{\partial \vec{x}}+\vec{a}^{s} \cdot \frac{\partial}{\partial \vec{v}}\right) f^{s}=0
$$

i.e., $f^{s}=$ constant

This means that the velocity distribution function is constant for an observer moving along a collisionless trajectory. Hence, the distribution function at every point along the trajectory can be obtained if it's value at one point (i.e., at the boundary) is given

\section{THE PLASMA SHEATH MODEL}

In the present work, we restrict ourselves to timeindependent, collisionless electrostatic problems, as is appropriate for the sheath regions. The $l d l v$ model of the plasma sheath is shown schematically in Fig. 1. The notation $l d l v$ indicates the fact that our model is one-dimensional both in configuration and in velocity space. The magnetic field B is normal to the wall and assumed to be "infinitely" strong, so that the $l d l v$ approximation is appropriate.

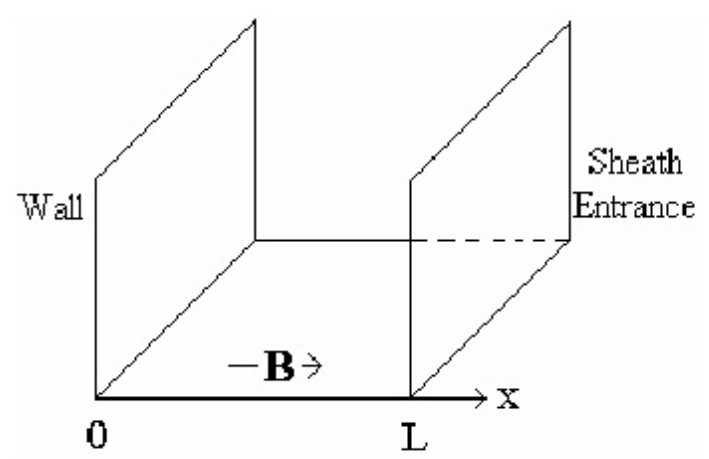

Fig. 1: $l d l v$ plasma she ath model.

The simulation region considered is bounded by two parallel planes located at $x=0$ and $x=L$, and the plasma is assumed to consist of electrons and one species of singly charged ions. The two boundaries are specified as follows. The right hand boundary $(x=L)$ is the "sheath entrance", separating the non-neutral, collisionless sheath region $(x<L)$ from the quasi-neutral, collisional presheath region $(x>L)$, where as the left-hand boundary $(x=0)$ represents an absorbing wall.

The potential value at $x=L$ is al ways chosen equal to zero, whereas the one at $x=0$ is fixed to a negative constant value, i.e., $\phi(x=0)=\phi_{0}=$ constant $<0$. We restrict ourselves to potential distributions $\phi(x)$ which decrease monotonically from $x=L$ to $x=0$ such that the electric field is always negative.

We assume that the plasma particles (electrons and ions) enter the simulation region from the right-hand boundary with cut-off Maxwellian velocity distribution functions, that the left-hand boundary does not emit any particles, and that both boundaries are perfectly absorbing. Accordingly, the electron velocity distribution function is given by,

$f^{e}(x, v)=A^{e} \exp \left[-\left(\frac{v}{v_{t}^{e}}\right)^{2}+\frac{e \phi(x)}{k T_{f}^{e}}\right] \Theta\left[v_{c}^{e}(x)-v\right]$

where

$v_{c}^{e}(x)=\sqrt{\frac{2 e\left[\phi(x)-\phi_{0}\right]}{m^{e}}}$

is the electron cut-off velocity at $x$. The ion velocity distribution function at $x=L$ is given by;

$f^{i}(L, v)=A^{i} \exp \left[-\left(\frac{\nu-v_{m L}^{i}}{v_{t j}^{i}}\right)^{2}\right] \Theta\left(v_{c L}^{i}-v\right)$

where 
$v_{t j}^{s}=\sqrt{\frac{2 k T_{f}^{s}}{m^{s}}}$

is the species-s thermal velocity, $v_{m L}^{i}$ is the ion "Maxwellianmaximum" velocity at $x=L$, and $v_{c L}^{i}$ (with $v_{c L}^{i}<0$ ) is the ion cut-off velocity at $x=L$. Here, $T_{f}^{e}$ and $T_{f}^{i}$ are the "formal" electron and ion temperatures.

Exact ion trajectories are followed, to calculate along them the ion distribution function, for the given ion injection distribution. The electrons, on the other hand, are assumed to have a half Maxwellian velocity distribution at injection, so that their density can be calculated analytically. Due to absorption at the wall, their full velocity distribution is a cutoff Maxwellian. Starting from an initial guess, the potential profile is iterated towards the final time-independent, selfconsistent state [6].

\section{RESULTS AND DISCUSSION}

Fig. 2 shows the self-consistent potential profile in the sheath region for different ion to electron temperature ratios at the sheath entrance. It shows very sharp gradient close to the wall but near the sheath entrance, the potential is seen to be almost constant. Hence, the major drop in potential is in the immediate neighborhood of the wall, as expected. Fig. 3 shows the variation of the wall potential for different values of presheath ion temperature. We see that on increasing the presheath ion temperature, the magnitude of the potential at the wall goes on decreasing. Fig. 4 shows the variation of charge density at wall for different values of presheath ion temperature. We see that on increasing the presheath ion temperature, the value of the charge density at the wall goes on increasing.

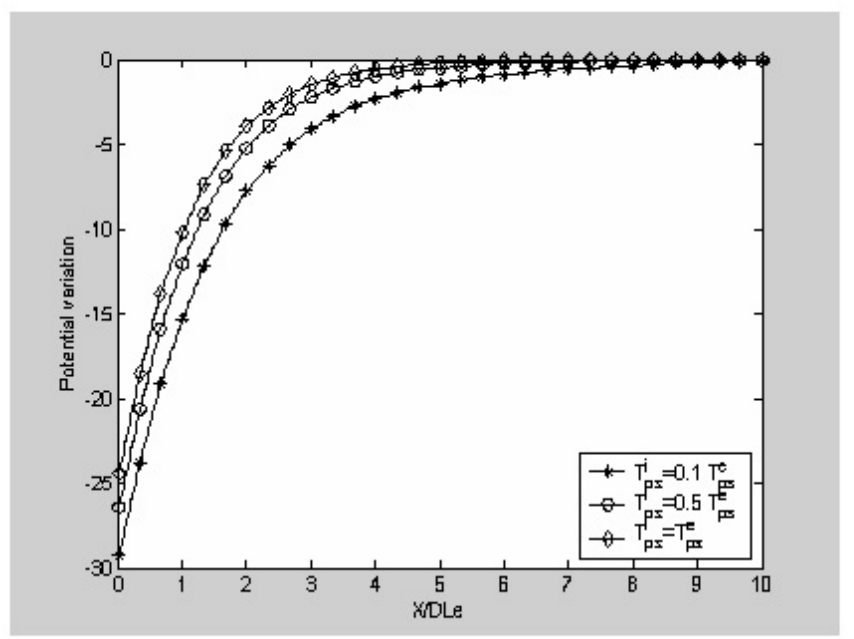

Fig. 2: Self consistent potential profile versus the distance from the wall for different presheath ion- electron temperature ratios.

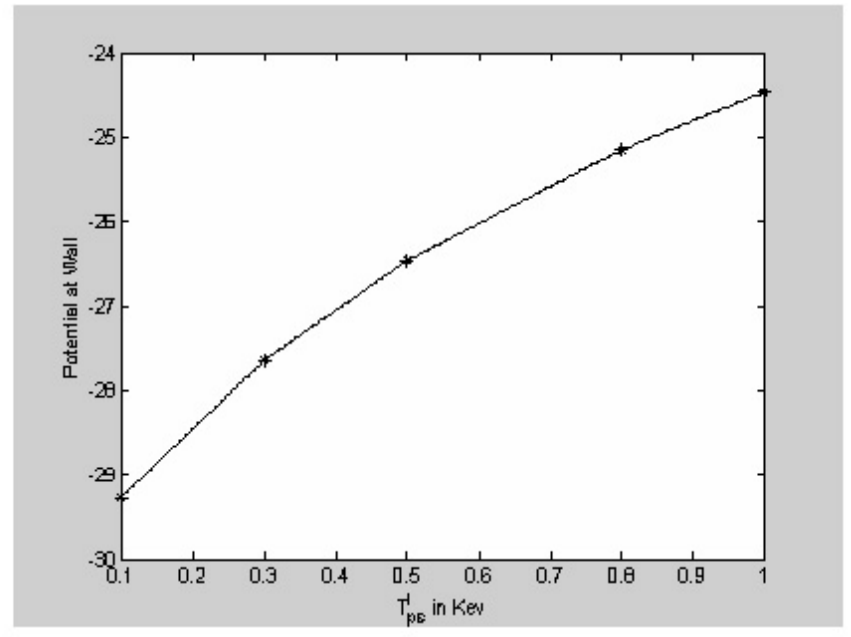

Fig. 3: Variation of potential at the wall for different presheath ion temperatures.

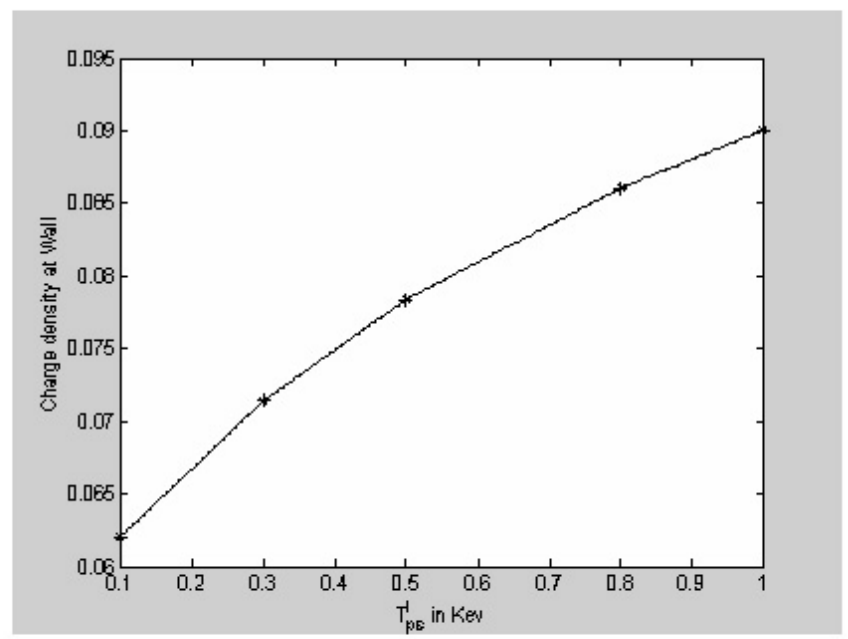

Fig. 4: Variation of charge density at wall for different presheath ion temperatures.

The above plots show that more ions reach the wall on increasing the presheath ion temperature. This is because more the presheath ion temperature more will be the ion thermal velocity and hence more ions reach the wall. Consequently, the electron density must also increase to maintain the constant potential at the wall. The ion density is usually one order higher than the electron density at the wall. This occurs because of reflection of electrons due to the negative potential at the wall. Thus the charge density in the sheath region is always positive with minimum value (i.e. zero) at the sheath entrance and maximum value at the wall. It is seen that the magnitude of the wall potential decreases on increasing the presheath ion temperature. This happens because, as we have stated earlier, more ions reach the wall for increased presheath ion temperature. In fact, the numbers of electrons also increase; however, the increment is more for ions than that for electrons. 
It has been found that, for the ratio of ion to electron presheath temperature equal to $0.1,0.5$ and 1 , the wall potentials were found to be $-29.2,-26.4$ and -24.4 volts. On taking the same ion to electron presheath temperature ratio, the electron and ion densities reaching the wall were $3.25 \times 10^{16}, 4.4 \times 10^{16}$ and $5.6 \times 10^{16} \mathrm{~m}^{-3}$; and $4.2 \times 10^{17}, 5.4 \times 10^{17}$ and $6.2 \times 10^{17} \mathrm{~m}^{-3}$ respectively although the corresponding values of both the ion and electron density at the sheath entrance was $10^{18} \mathrm{~m}^{-3}$.

The applied kinetic model is expected to give better insight to the sheath formation. This work may be extended to higher dimensional analysis including the effect of oblique magnetic field and to study the effect of varying other parameters, etc.

\section{REFERENCES}

[1] Bohm, D. 1949. The characteristics of electrical discharges in magnetic fields, Edited by A. Guthry and P.K. Wakerling, McGraw Hill, 77

[2] Riemann, K.U. 1991. J. Phys. D. Appl. Phys. 24: 493.

[3] Riemann, K. U. 2000. J. Tech. Phys. (Special Issue). 41(1): 89.

[4] Khanal, R. 2003. A kinetic trajectory simulation model for bounded plasmas, $\mathrm{PhD}$ Thesis, University of Innsbruck, Austria.

[5] Joshi, R. etal. 2006. Ann. Proc. of Nepal Physical Society 22 (No 1, Special Issue): 37.

[6] Bhattarai, S. K. 2006. Effect on the plasma sheath structure due to the temperature variation of the in-streaming particles, M. Sc. Diss ertation, Tribhuvan University, Kirtipur, Kathmandu, Nepal. 